\title{
SOBRE LA CONCEPCIÓN BERGSONIANA DE "INTUICIÓN" Y LAS CONSECUENCIAS PARA LA COMPRENSIÓN DE LA CIENCIA Y LA METAFÍSICA (UNA COMPARACIÓN CON KANT)
}

Ruth Gordillo R. ${ }^{1}$

\section{RESUMEN}

El filósofo francés Henri Bergson señala una importante crítica a Kant desde la formulación del concepto de intuición, fundamental en la constitución de la teoría kantiana. Este artículo parte de la definición bergsoniana de dicho concepto además del de duración como condición de posibilidad para el conocimiento. La comparación con la definición de intuición sensible del kantismo y las consecuencias que dicha comparación supone para la compresión de ciencia y metafísica, serán el objeto central de este trabajo.

Palabras clave: intuición, evolución, conocimiento, ciencia, metafísica

\section{ABSTRACT}

French philosopher Henri Bergson formulates an important critique against Kant using the concept of intuition, an essential element in Kantian epistemology. This article considers the bergsonian definition of the concept of intuition in addition to duration as a necessary condition for knowledge. The central aim of this article is a comparison of the definition of sensible intuition in Kantian philosophy and its consequences for the understanding of science and metaphysics.

Key words: intuition, evolution, knowledge, science, metaphysics

\footnotetext{
1 Pontificia Universidad Católica del Ecuador, Facultad Eclesiástica de Ciencias Filosófico-Teológicas, Escuela de Filosofía, Quito, Ecuador (rgordillo@puce.edu.ec).
} 
Es relativo el conocimiento simbólico por conceptos preexistentes, que va de lo fijo a lo moviente pero no el conocimiento intuitivo, que se instala en lo moviente y adopta la vida misma de las cosas.

Esta intuición alcanza un absoluto.

H. Bergson

\section{INTRODUCCIÓN}

La delimitación del tema de este ensayo surge de la relación, muy trabajada ya a lo largo de la historia de la filosofía del s. XX, entre Kant y Bergson. Las posturas más ampliamente aceptadas por los herederos de Kant dentro del neopositivismo lógico y matematicista se pueden resumir en la crítica de Bertrand Russell, especialmente formuladas en la Historia de la filosofía occidental (1946), en el capítulo correspondiente a Bergson, donde entiende que su filosofía contiene un aspecto negativo muy importante, cual es, el carácter no intelectualista, carácter que le lleva a desarrollar una serie de tesis sobre el conocimiento humano y la ciencias, plagadas de errores. Sin embargo, es innegable el significado del filósofo francés en lo que tiene que ver con las consideraciones fundamentales respecto de la condición del sujeto en los actos cognitivos que, además de los aspectos atinentes al funcionamiento del cerebro, admite procesos importantes que permitieron la consolidación de tendencias y teorías en el siglo XX, especialmente en autores franceses posestructuralistas. En este contexto, el ensayo de Maurice Merleau-
Ponty, "Bergson haciéndose" (1960), da cuenta de esta influencia y, por ello, servirá para determinar las relaciones que se establecen en el proceso cognitivo bergsoniano y que enlazan los conceptos de metafísica y ciencia a partir de la noción de intuición; en la misma perspectiva, la comparación con Kant -que de suyo resulta obvia- completará el propósito de este ensayo. Esta relación entre los dos autores no es nueva y ha dado lugar a innumerables textos, quizás por ello la vigencia de las consecuencias e implicaciones preña una parte importante de las teorías filosóficas contemporáneas que se desarrollan en torno a sujeto y a sus problemáticas.

Además, es necesario hacer referencia a la crítica al kantismo que realiza Bergson para entender el punto de partida de las formulaciones que construye en torno a los procesos del conocimiento y, además, para entender cómo, desde ellas, concibe tanto a la metafísica como a la ciencia. En este sentido, la tesis de este ensayo se define en los siguientes términos: Bergson determina una búsqueda respecto del acto de la 
cognición más allá de la fundamentación kantiana que parte de la formulación del a-priori, en esta búsqueda, la intuición se concibe de manera diferente en los dos autores, al igual que las consideraciones sobre la metafísica y los corolarios para la ciencia. El desarrollo de esta afirmación supondrá abordar el concepto de intuición bergsoniano que remite al de duración y que será trabajado con relación a la categoría de intuición del kantismo, a partir de ello será posible marcar los límites entre los dos filósofos con relación a las concepciones de ciencia y de metafísica.

\section{INTUICIÓN Y DURACIÓN}

La definición de duración de Bergson y las categorías a-priori del entendimiento de Kant marcan una diferencia fundamental en la posibilidad y naturaleza del conocimiento que, en primera instancia, remite a la explicación que el filósofo francés busca respecto del origen del a-priori, aspecto que no está planteado en Kant. En sentido bergsoniano, una vez que se supera la generalización que el lenguaje realiza a partir de las percepciones, es posible acceder a los datos inmediatos de la conciencia.

El primer concepto bergsoniano que deviene fundamental para el trabajo de este punto es el de intuición. Hay que diferenciar percepción de intuición de la realidad como tal; esta categoría distingue entre la "la unidad en la que se piensa"y"la unidad convertida en cosa" después de ser pensada (Bergson, 2011: 62). Tal diferencia no da cuenta de dos formas de concebir de la conciencia, sino de dos estados de la conciencia; en esta medida, el primer problema que aparece es el de la conformación de la unidad con relación a la multiplicidad; dilucidar la forma en que cada una se constituye es el punto nodal de su reflexión, de allí la cuidadosa explicación de la definición de número. De esta explicación, es importante destacar lo siguiente: Bergson (2011, págs. 60 - 61) dice que al pensar la unidad aisladamente se piensa en la indivisibilidad pero, cuando se pasa a la siguiente unidad, ella se objetiva, se hace cosa y, obviamente, se piensa la multiplicidad. Ahora bien, ¿qué es lo que se pone de manifiesto en este paso de una unidad a otra? No otra cosa que la posibilidad de determinar los múltiples estados de la conciencia que, además, explican las diversas formas de concebir el mundo y, al mismo tiempo, de concebir el proceso interno de la conciencia. Desde la exposición de concepto de número, pasa a la exposición de la intuición de los objetos materiales. La condición epistemológica para ello se da tanto en la multiplicidad del mundo de las cosas como en la mul- 
tiplicidad de los hechos de la conciencia. La diferencia con la conceptualización de los objetos materiales, que remiten a un número, radica en que los hechos de la conciencia, es decir, los hechos internos, no pueden utilizar unidades -números- sin antes haber realizado una representación simbólica (2011: 65). El aporte de Bergson se entiende en el intento de profundizar en los procesos de la conciencia como ámbito del conocimiento, en esta perspectiva, considera una complejidad que no se resuelve, como en Kant, apelando a los límites de la razón pura respecto de posibilidad del conocimiento, sino desde el trabajo de los conceptos de espacio y tiempo. Vale decir que estos conceptos no son considerados categorías a-priori, al menos no en el mismo sentido de la filosofía kantiana.

Para Kant (2006:1 17), la intuición se define como la relación inmediata del conocimiento con el objeto; sin embargo, la intuición está ligada a la sensibilidad que es la capacidad de receptividad de un objeto que, además, afecta al sujeto. La intuición sensible es la categoría kantiana que define la forma en que el sujeto conoce los datos que provienen del objeto, el resultado de este proceso es el fenómeno compuesto de materia -aquello que proviene de la sensación y que se da a-posteriori- y, de forma - dada a-priori, por tanto, presente en toda sensación-. Es determinante la naturaleza a-priori de las categorías de espacio y tiempo que constituyen la condición de posibilidad de la intuición sensible. En las Sección primera de la Estética trascendental de la Crítica de la razón pura, Kant expone las características a-priori de las dos categorías; de la exposición surge el carácter lógico del apriori en términos generales en la medida que espacio y tiempo tienen una función ordenadora de las percepciones de los sentidos, entiéndase en el mismo sentido, y con más fuerza, la formulación de las doce categorías del entendimiento que hacen posible la segunda representación que se opera sobre el fenómeno -definido por Kant como la primera representación que surge de la intuición sensible y de los datos que ella capta del objeto-. Quiere decir que la condición del proceso de conocimiento se rige, fundamentalmente, por estructuras lógicas, propias de la racionalidad inapelable del sujeto. De igual forma, para Bergson, hay una exigencia lógica en la aceptación de ciertas propiedades del mundo físico; en el Essai sur les donnés immédiates de la conscience (1889), respecto de la impenetrabilidad de los cuerpos dice, "...une propieté de ce genre, purement négaive, ne saurait nous être révélée par les sens..."2 (2011: 65), entonces ¿de dónde surge la certeza

2 "...una propiedad de este género, puramente negativa, no podría sernos revelada por los sentidos..." (Bergson, 1999: 69). 
de esta creencia? Obviamente es una necesidad lógica, concluirá más adelante. La cuestión se vuelve sobre la necesidad de marcar la diferencia entre Kant y Bergson.

Para Kant, las categorías a-priori, tanto de la intuición sensible como del entendimiento, son condiciones estructurales que condicionan y posibilitan el conocimiento, sea del mundo físico, sea del mundo de los objetos ideales. Bergson asume que, al admitir la impenetrabilidad de los cuerpos como propiedad del mundo físico, se ha reconocido "la solidarité des notions de nombre et d'espace", lo que equivale a "énoncer une propiété du nombre, pultôt que de la matière."3 (2011: 66) Esta misma forma de comprensión la extiende a las cosas de la conciencia pero, señala, que ellas requieren primero, de una representación simbólica en el espacio que, en términos de la comparación que se ha formulado entre los dos autores, es imposible en Kant. En las dos formulaciones, el proceso corresponde al sujeto y, en términos amplios, no al mundo físico. Subsiste, además, el interés, tanto de uno como de otro filósofos, por explicar cómo es posible conocerse a sí mismo. Para Kant, en el ámbito de la razón pura, ello significa conocer los procesos del entendimiento, procesos que llama psíquicos o internos. En el $\S 6$ de la Crítica de la razón pura (1787) bajo el título "Consecuencias de estos conceptos"-se refiere a la exposición de los conceptos de espacio y de tiempo-, dice:

b) El tiempo no es otra cosa que la forma del sentido interno, esto es, del intuirnos a nosotros mismos y nuestro estado interno. Pues el tiempo no puede ser una determinación de fenómenos externos. No se refiere ni a una figura ni a una posición, etc., sino que determina la relación entre las representaciones existentes en nuestro estado interior (2006: 76-77).

Por detrás de la capacidad de representación que poseen los sujetos, está, para Bergson, una comprensión dualista de la materia: Io físico y lo psíquico; sin embargo, los dos elementos están relacionados.

La explicación de la opción bergsoniana para explicar la dualidad materia-psiquismo, desborda el proceso del conocimiento. Quizás el libro Matière et mémoire (1896), Bergson da pistas sobre una sui generis comprensión del dualismo que subsiste en su filosofía y que, para B. Russell, valió para considerarlo irracionalista. El asunto no es discutir si Bergson es o no irracionalista, sino entender qué significado tiene una postura que marcó la historia de la filosofía en el s. XX. De allí podrá definirse su distancia

\footnotetext{
3 "la solidaridad de las nociones de número y de espacio"... "enunciar una propiedad del número, más que de la materia." (Bergson, 1999: 70).
} 
con las concepciones y posturas respecto de la ciencia y, por otro lado, los posibles aportes a la filosofía francesa. La crítica de Russell sostiene, de manera particular, la explicación del sentido que para Bergson tiene el concepto de evolución, utilizado para definir tanto los procesos del conocimiento como para fundamentar su postura espiritualista que, para el filósofo inglés, está acompañada de un irracionalismo poco aceptable para quien se refiere a la ciencia; queda claro que la medida para decidir sobre Bergson será la ciencia y su imponente corpus.

Ahora bien, Bergson entiende que la realidad del sujeto es de naturaleza psicológica, de manera que cada vez el estado interno del sujeto puede cambiar; ese cambio no se reduce a formas biológicas, sino que está sujeto a un impulso que permite que el cuerpo siga en movimiento; en tal sentido la evolución es evolución creadora. En la obra L'évolution créatrice (1907), Bergson señala que

...la théorie de la connaissance et la théorie de la vie nous paraissent inséparables l'une de!'autre. Une théorie de la vie qui ne s'accompagne pas d'une critique de la connaissance est obligée d'accepter, tels quels, les concepts que l'entendement met à sa disposition : elle ne peut qu'enfermer les faits, de gré ou de force, dans des cadres préexistants qu'elle considère comme définitifs. ${ }^{4}$ (2009, p.9).

Quiere decir que el conocimiento no produce un saber en el sentido tradicional, esto es, el conocimiento del mundo exterior a partir de ciertas estructuras puramente racionales del sujeto, sino que busca dar cuenta de una realidad más rica cuya profundidad solo puede ser producto de un acto de creación. Este acto será el punto de escisión de la filosofía bergsoniana con el kantismo. Lo que resta, en este punto, es configurar los límites de la evolución creadora, como parámetro de la crítica a la concepción racionalista de Kant; en este contexto, la crítica de Russell tiene gran importancia, aun cuando es posible señalar que lejos del irracionalismo, Bergson determina la posibilidad de entender cómo el sujeto se constituye en el acto de conocer, no solamente desde la inteligencia, sino desde aspectos profundos, postulados como psíquicos. De esta manera, el dualismo de la tradición racionalista se corrige, ya no es más res cogitans y res extensa, sino vida -que se entiende como cuerpo y psiquismo-y materia. El replanteamiento tendrá otro tipo de consecuencias en la concepción del sujeto pero, fundamentalmente, otros supuestos tanto epistémicos como metafísicos.

\footnotetext{
${ }^{4}$... la teoría del conocimiento y la teoría de la vida nos parecen inseparables una de otra. Una teoría de la vida que no se acompañe de una crítica del conocimiento está obligada a aceptar, al pie de la letra, los conceptos que el entendimiento pone a su disposición: no puede sino encerrar los hechos, de grado o por fuerza, en cuadros preexistentes que considera como definitivos (Bergson, 1963: 436-437).
} 
Para determinar estos supuestos es necesario remitirse a la definición de evolución : "L'évolution doit donc comporter à tout moment une interprétation psychologique quien est, de notre point de vue, la meilleure explication, mais cette explication n'a de valeur et même de signification que dans le sens rétroactif. $»^{5}$ (Bergson, 2003: 39). La vida concebida con relación a esta forma de entender la evolución, es, sin lugar a dudas, una vida que desborda la teleología de la tradición filosófica moderna accesible solamente desde la racionalidad de la ciencia positiva; la inteligencia se comprende como el resultado de "...l'évolution au cours du trajet... II faut substituer à l'intelligence proprement dite la réalité plus compréhensive dont lintelligence nést que le rétrécissement." ${ }^{\prime 6}$ (2003: 39-40). El supuesto de realidad es sui generis, parecería integrar el sujeto, en tanto vida e inteligencia, al mundo exterior; tal forma de integración, que no es la única en la filosofía, implica la relación del sujeto con el mundo en el tiempo y en el espacio. Cuando Bergson aborda esta relación, aparecen claramente las notas que caracterizarán su postura frente a las del
Kant y del positivismo lógico desde el cual escribe Russell. Asimismo, bosquejará los aportes a la filosofía francesa del s. XX en los términos que se sustraen a las concepciones tradicionales y que se ubican en el camino del desgarramiento formulado por Merleau-Ponty en el texto sobre Bergson.

Russell (1946: 820) sostiene que para Bergson,

Evolution is not primarily explicable by adaptation to environ meat; adaptation explains only the turns and twists of evolution, like the windings of a road approaching a town through hilly country. But this simile is not quite adequate; there is no town definite goal, at the end the road along which evolution travels. ${ }^{\text {? }}$

El concepto de evolución así entendido, se distancia de la posibilidad de seguir la pista de un proceso cuya ley fundamental es la adaptación al medio; aún más, la ausencia de una finalidad que lleve a algo esencialmente nuevo en el mundo, concluye Russell; quiere decir que Bergson se ubica en una com-

\footnotetext{
5 "La evolución - dice Bergson- debe, pues, implicar en todo momento una interpretación psicológica, que es, desde nuestro punto de vista, la mejor explicación, aunque esta explicación no tiene valor ni incluso significación más que en el sentido retroactivo." (Bergson, 1963: 482).

6"... evolución en el curso de su trayectoria... Es preciso sustituir la inteligencia propiamente dicha por la realidad más comprensiva, de la cual la inteligencia no es más que su reducción." (Bergson, 1963: 482-483).

7 "La evolución no es primordialmente explicable por la adaptación al medio; la adaptación explica solamente los giros y vueltas de la evolución, como las vueltas de una carretera que se aproxima a una ciudad atravesando un campo montañoso. Pero este símil no es del todo adecuado; no hay una ciudad, una meta definidas al final del camino por donde marcha la evolución."
} 
prensión mecanicista y teleológica que, sumada a la categoría de creación, resulta extraña a la ciencia; queda asumir un pensamiento creador, tal y como lo hacen los artistas: "As against both these views, though with more sympathy for teleology than for mechanism, Bergson maintains that evolution is truly creative, like the work of an artist."8 (Russell, 1946: 820) El término sobre el que Russell regresa para desarrollar el análisis, es el de trabajo de un artista. ¿Qué tiene de problemática esta tesis? Basta recordar el impacto que propuestas como la de Nietzsche y Heidegger tuvieron en la filosofía de la ciencia de corte positivista. La reacción de Russell resulta, por decirlo de algún modo, natural. Sin embargo, los supuestos epistémicos son distintos, Bergson asume una concepción de la realidad que no es ajena a la voluntad, hay un carácter desiderativo en la conformación de la realidad desde el conocimiento; lo que llama vital está dado en relación a lo querido (2003: 175). Si bien puede pensarse que esto querido, ligado a la voluntad (no entendida en términos kantianos, sino más bien relacionada con aspectos profundos del sujeto no totalmente definibles), da cuenta de una postura irracional, es importante considerar que en la concepción bergsoniana están presentes elementos provenientes, no exclusivamente de las ciencias, sino de teorías como el psicoanálisis, presente en la época con gran fuerza. ${ }^{9}$ El trabajo de profundización en las estructuras del sujeto, deja una impronta en la filosofía de Bergson que es reconocida por filósofos como Merleau-Ponty: (1964: 224)

Las conciliaciones, las celebraciones,
no tendrían que hacernos olvidar el
camino que Bergson, solo, trazó y del
cual nunca renegó, esta manera di-
recta, sobria, inmediata, insólita, de re-
hacer la filosofía, de buscar lo profun-
do en la apariencia y lo absoluto más
allá de nuestros ojos, en fin, con los
mejores modos, el espíritu de descu-
brimiento que es la fuente primera del
bergsonismo.

El trabajo del filósofo francés sería, de acuerdo con Merleau-Ponty, una suer-

\footnotetext{
8 "Frente a ambas miradas, pero con más simpatía por la teleología que por el mecanicismo, Bergson mantiene que la evolución es verdaderamente creativa, como el trabajo de un artista."

${ }^{9}$ La discusión que se ha mantenido sobre la posible relación entre estos autores parece decidir que, primero, hay elementos comunes en los estudios que les llevaron a hacer sus respectivas propuestas, por ejemplo, los estudios sobre las afasias presentes en la formulación de la teoría freudiana de manera fundamental -en el texto de 1891 Las afasias-y que le sirvió para diferenciar a los neuróticos con sintomatología pero sin daño neurológico de pacientes que presentaban afasias a partir de un daño anatómico. De igual modo, en la obra de Bergson el tema aparece especialmente en Materia y memoria, texto en el que, además, admite el inconsciente, aunque, es necesario aclarar, no tiene el mismo sentido que en el freudismo; segundo, hay que entender que el trabajo de Bergson es fundamentalmente filosófico, no así el de Freud cuyo interés está dado en el campo de lo psíquico desde una perspectiva positivista que se alejaba de las consideraciones metafísicas.
} 
te de apertura hacia un horizonte del todo nuevo que reformula los parámetros de la filosofía desde el análisis de "los principios de mecánica de los que se servía sin rigor Spencer"10 (1964: 225); el resultado de este punto de partida lo llevó a la formulación del concepto de duración que concibe de manera distinta al tiempo. Antes de abordar este concepto queda por definir lo que, con relación a lo tratado en estas páginas, entenderá por intuición, de esta definición será posible deducir la posibilidad de la metafísica y los límites de la ciencia.

El carácter psicologista que surge de la comprensión bergsoniana del proceso del conocimiento, habla de una intuición no intelectual, a diferencia de Kant; esta tesis implica una compleja estructura explicable desde consideraciones fisiológicas y psíquicas, vinculadas a la forma en que la filosofía ha tratado los procesos de la percepción. En la conferencia "La percepción del cambio" dictada en 1911, en Cambridge, dice que el filosofar se aparta de la acción para especular, para trabajar con conceptos; en el campo de la percepción de las cosas, en tanto quedan aspectos sin explicarse, los filósofos se han visto obligados a"... compléter la perception par la conception, -celle-ci devant combler les intervalles entre les données des sens ou de la conscience et, par là, unifier et systématiser notre connaissance des choses."11 (Bergson, 1969: 83). La explicación bergsoniana tomará estos aspectos con el fin de profundizar en el conocimiento que está limitado por los datos de lo que denomina, las percepciones naturales; este será el campo propio de la filosofía, más cercano a las artes que a las ciencias. ¿En qué sentido?, en tanto el artista es capaz de "extender las facultades de percibir"; aun sin saberlo puede dar cuenta de una realidad que está reducida por la percepción corriente más bien ligada a una cierta practicidad (Bergson, 1969: 84 - 85). Esta forma profunda de percibir remite a una comprensión más completa de la realidad definida como intuición solamente en la medida que se liga a la duración interior, es decir "a la visión directa del espíritu por el espíritu" (1969: 19). Aun antes de entrar en la explicación y análisis del concepto de duración, la diferencia con Kant es notable. Aquello que Bergson niega a la intuición, en tanto intelectual, define la condición de posibilidad del conocimiento para Kant. La diferencia

\footnotetext{
${ }^{10}$ Para fines del s. XIX, la importancia del mecanicismo en la perspectiva teórica de Spencer, radicó en la necesidad de explicar, desde las leyes de la ciencia natural, los procesos humanos, biológicos, psíquicos y sociales. El darwinismo social que se le atribuye, surgió de temas similares a los tratados por Bergson. El interés fue el mismo pero los supuestos no.

11 “...completar la percepción por la concepción, debiendo esta colmar los intervalos entre los datos de los sentidos o de la conciencia, y con ello unificar nuestro conocimiento de las cosas." (Bergson, 1972: 109).
} 
reside en el supuesto de realidad del que parte Bergson, para él, la realidad es movimiento y la intuición lo capta; desde esta forma de comprensión -no solo la filosofía kantiana, sino la de Platón y, por extensión en gran medida la filosofía occidental-, la realidad se halla atrapada en lo inmóvil; de allí que la intuición, tal como la formula el filósofo de Königsberg, sea puramente intelectual, aun cuando esté ligada a la sensibilidad. El concepto de intuición, dentro del sistema bergsoniano, terminará de consolidarse con relación al de duración.

El segundo concepto determinante en el sistema bergsoniano es el de duración que, en términos generales, tiene, al menos, dos formas de entenderse, como duración pura, que es inextensa con relación al tiempo -considerado de manera sui generis, como se verá más adelante--, y como duración ligada al espacio. Para hacer la distinción entre ellas, Bergson se remite al concepto de número y a su proceso de constitución; la razón que funda la elección de este concepto en particular tiene quever con que "... il devient aisé de faire la part exacte du sujectif et de l'objectif dans l'idée de nombre."12 (2011: 63) En función del interés de este artículo, subjetivo y objetivo son términos que permitirán desarrollar la relación entre la perspectiva del filósofo francés y la de Kant respecto de la postura de cada uno con relación a la ciencia y su delimitación de la metafísica; en el mismo orden, lo que se afirma para el concepto de número, permitirá distinguir a los conceptos referidos a objetos de la experiencia sensible, a partir de la distinta concepción de la multiplicidad, tema que ya se ha abordado con relación al concepto de intuición pero que requiere ser precisado. Ahora bien, en el proceso que permite formular el concepto de número se distinguen dos formas de concebir la unidad: la primera es provisional y la segunda es definitiva; aquélla reside en la multiplicidad constitutiva de la unidad de la representación producto de lo que llama el "acto simple de la percepción" de la inteligencia; esta se formará "añadiéndose a sí misma" (Bergson, 2011:60). El problema que ve el autor en la provisionalidad de la representación de la unidad, en este caso remitida al concepto de número, exige entender lo que implica la forma simple de concebir de la mente; sin embargo, la posibilidad de la representación de lo real, esto es, referida a la duración, halla su sustento en la provisionalidad que permite mantener la divisibilidad de las unidades que conforman un número cualquiera; la unidad entendida como definitiva, en cambio, concluye en la indivisibilidad. Este asunto, sin duda complejo, le lleva a trabajar el proceso de elaboración de

12 “. .. resulta fácil el cómputo de lo subjetivo y de lo objetivo en la idea de número." (Bergson, 1999, pág. 67). 
los conceptos que no tienen un sustrato en la materialidad. Estos serán los que se revisarán más tarde con relación a la concepción kantiana; las conclusiones de esta comparación, permitirán delimitar el sentido y alcance del concepto de duración bergsoniano en el orden de la comprensión del concepto de intuición.

Bergson sostiene que las cosas deben percibirse por la duración. En el texto citado en el párrafo anterior, el autor considera que la posibilidad de la representación que realiza la conciencia reside en el carácter aislado de las impresiones que son captadas por los sentidos; en esta medida la representación consiste en la constitución de una unidad a partir de la yuxtaposición de los elementos distintos (2011: 56). La concepción de la multiplicidad que se había explicitado para el caso del concepto de número va a encontrar dos determinaciones en tanto, «Quand nous affirmons que le nombre est un, nous entendons par là que nous le représentons dans sa totalité par une intuition simple et indivisible de l'esprit: cette unité renferme donc une multiplicité, puisque c'est l'unité d'un tout». ${ }^{13}$ (2011: 59-60 ) El asunto que aquí interesa remite a la manera de concebir la intuición; en efecto, la intuición, una vez ligada a la duración, puede captar el mo- vimiento como continuidad que trae siempre algo nuevo; una vez establecida la relación intuición-duración, Bergson asume que la intuición, a partir de la percepción de la continuidad, «...elle voit, elle sait que l'esprit tire de lui-même plus qu'il n'a, que la spiritualité consiste en cela même, et que la réalité, imprégnée d'esprit, est création." ${ }^{14}$ (1969: 21) Ahora bien, el espíritu, en tanto actúa sobre sí mismo e impregna a la realidad con su naturaleza, se constituye en la condición para afirmar que la realidad es creación del espíritu a través de la intuición ligada a la duración. Las consecuencias de esta forma de concebir el proceso de la percepción, superado por una intuición creadora de realidad, determinan el alejamiento de la perspectiva kantiana; aun más, dan cuenta de una doble definición de intuición, intuición intelectual e intuición creadora. Esta última permite desvelar el cambio, es decir, el tiempo, que se halla cubierto por el espacio y por las percepciones que se dan en él. Estas percepciones desembocan en las conceptualizaciones producto de un acto del intelecto puro, como en el caso de Kant; quedan, en esta medida, alejadas de la realidad temporal que es el objeto de la búsqueda bergsoniana y que se define como, "...la création continue d'imprévisible nouveauté qui semble se

13 "Cuando afirmamos que el número es uno, entendemos por ello que nos lo representamos en su totalidad por una intuición simple e indivisible de la mente: esta unidad encierra, pues, una multiplicidad, porque es la unidad de un todo." (Bergson, 1999: 64).

14 "... ve y sabe que el espíritu extrae de sí mismo más de lo que tiene, que la espiritualidad consiste en eso mismo, y que la realidad, impregnada de espíritu, es creación." (Bergson, 1972: 33). 
poursuivre dans l'univers."15 (Bergson, 1969: 56).

Desde esta perspectiva, la realidad está constituida por materia y espíritu, parecería que este espíritu está en el sujeto que conoce y que, en cuanto material, forma parte del universo. Ahora, este ser vivo, que es el hombre, « ... dure essentiellement; il dure, justement parce qu'il élabore sans cesse du nouveau et parce qu'il n'y a pas d'élaboration sans recherche, pas de recherche sans tâtonnement. $»^{16}$ (Bergson, 1969: 57). Hay una conciencia que está impelida a la indagación que no solamente encuentra algo ya dado en la realidad, sino que la constituye fundamentalmente. De esta formulación surge el sentido del concepto de duración y, de forma consistente con él, la definición de cambio en términos de evolución creadora.

Veamos, si la duración aparece ligada a la temporalidad, la concepción de espacio, en la perspectiva kantiana, ha contribuido, según Bergson, a sustentar una forma de percepción que impide llegar a la realidad en su constitución verdadera. En el apartado de la Crítica de la razón pura, "Estética trascendental", anota el filósofo francés, el espacio tiene consistencia propia independiente- mente de su contenido (2011: 69-70). ¿Qué se sigue de esta concepción? Podría decirse que un olvido del análisis de la naturaleza del espacio, olvido que toca a las ciencias de manera directa y que desemboca en el empirismo o en el nativismo. El corolario de estas posturas aleja el trabajo, tanto filosófico como científico, de la búsqueda de la realidad en cuanto movimiento. Aun cuando hay intentos por reintegrar el espacio a los contenidos, estos, no se han alejado del presupuesto kantiano de la sensibilidad trabajando con categorías a-priori (2011: 70-71). Vale decir que la comprensión de la realidad como movimiento, solo será posible si se logra integrar el espacio al proceso de percepción del movimiento donde hay que"... distinguer entre la perception de l'étendue et la conception de

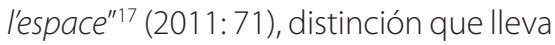
a abandonar la idea de un espacio homogéneo, resultado de un acto de la inteligencia $y$, por tanto, reducible al espacio de la geometría. Bergson, en cambio, en términos de la duración, sostendrá que la percepción del cambio, en el espacio integrado al tiempo, se dificulta porque la duración no solo se da en el sujeto sino en el mundo exterior; tal dificultad responde a la forma de percepción mediada por el lenguaje -que espesa el velo y esconde el movi-

\footnotetext{
15 "... la creación continua de imprevisible novedad que parece proseguirse en el universo." (Bergson, 1972: 89).

16 "... dura esencialmente: dura justamente porque elabora sin cesar algo nuevo y porque no existe elaboración sin búsqueda, ni búsqueda sin tanteos." (Bergson, 1972: 89).

17 "... distinguir entre la percepción de la extensión y la percepción del espacio..." (Bergson, 1999: 74).
} 
miento- y, además, por la acción de la inteligencia. La propuesta de la filosofía bergsoniana es ir más profundo en la búsqueda de la realidad donde el cambio se entiende como un conjunto de estados tanto de las cosas como de la conciencia percipiente. La duración es duración-cualidad determinada por momentos heterogéneos, pero no divisibles, que subyace a la percepción de estados homogéneos surgidos de la concepción de un espacio también homogéneo y desligado de sus contenidos, dentro del cual es posible la división de momentos y estados. La distancia con Kant es evidente, Russell la recoge cuando señala que Bergson no distingue "... the act of knowing and the object known..." aún más, "The distinction which Bergson has in mind in the above is not, I think, the distinction between the imaging as a mental occurrence and the thing images as an object."18 (1946: 836). Quiere decir que Bergson, efectivamente, no parte de la distinción entre sujeto y objeto que realiza el racionalismo ilustrado y que está presente en
Kant; al contrario, como dice MerleauPonty, Bergson procura un saber absoluto de lo absoluto pero de manera extraña pues, "no conocemos todos nuestros recuerdos, ni tampoco toda la densidad de nuestro presente", el contacto con uno mismo es, según Bergson, "coincidencia parcial". Luego, el saber absoluto es inherente al yo. A diferencia del racionalismo, de corte kantiano, MerleauPonty (1964: 225) sostiene que lo novedoso es dar a la filosofía no un yo pienso dentro de la inmanencia, sino "un ser uno mismo" cohesionado aunque, al mismo tiempo, desgarrado. Aquí radica la herencia que asumirá, en gran medida, la filosofía francesa a partir de la década de los 60.

Queda, entonces, retomar la segunda parte de este trabajo, esto es, la delimitación entre ciencia y metafísica, como resultado de las consideraciones que Bergson hace respecto del proceso de conocimiento del mundo; hacerlo implicará poner en relación las tesis de Bergson y las de Kant.

\section{CONSECUENCIAS: CIENCIA Y METAFÍSICA}

Antes de abordar este último apartado, vale señalar que en la filosofía de Bergson se pueden diferenciar dos mo- mentos respecto de la crítica a Kant. En lo que sigue, el texto fundamental para determinar la postura del francés, será $L a$

\footnotetext{
18 ". . el acto del conocimiento y el objeto conocido..." "La distinción que Bergson tiene en mente aquí, no es, yo pienso, la distinción entre el imaginar como suceso mental y la cosa imaginada como objeto."
} 
introducción a la metafísica (1903). Fréderic Worms, (2001) dice que la relación entre los dos autores tiene su fundamento en el problema del entendimiento que, por otra parte, sustenta la conformación del concepto de intuición, vale aclarar. La tesis de Worms se resume en lo siguiente: primero, Bergson asume la independencia del entendimiento en el proceso del conocimiento, independencia formulada por Kant; segundo, esta independencia no es total para el filósofo francés, de serlo, se abriría una diferencia insalvable entre el pensamiento y la realidad; $y$, finalmente, el límite de la independencia del entendimiento, se desplaza, en la filosofía bergsoniana hacia una comprensión que dispara la diferencia entre la forma de entender la intuición y la metafísica que cada uno postula (2001: 461). En este contexto, al poner a Bergson y Kant frente a frente para determinar la distancia que los separa, es lícito anotar que la comparación se recoge en este artículo, parte del texto sobre la metafísica (1903). Sin embargo, no serán abordados ni L'Énergie espirituelle (1919) ni Les deux sources de la morale et de la religión (1932); esta aclaración es necesaria en el orden otras lecturas que profundizan en la relación que nos ocupa.

La intención de Bergson es delimi- tar la metafísica desde la ciencia de la conciencia, en este enunciado se marca la diferencia con Kant; la pregunta obvia es, ¿en qué medida la ciencia de la conciencia, como la concibe Bergson, se aleja de la concepción kantiana de ciencia? La respuesta determinará la concepción de metafísica de cada uno de los autores. El punto de partida del filósofo francés es la forma en que la conciencia percibe el movimiento real, es decir, la duración. En el apartado anterior se ha definido el concepto de duración, en esta segunda parte, se trabajará a partir del ensayo aparecido en 1903 en la Revue de Métaphysique et de Morale, titulado "Introducción a la metafísica"; en el Prólogo de este ensayo, Bergson parte de la necesidad de plantear un giro en las concepciones del criticismo kantiano y del dogmatismo que dominaban en esos momentos: "Depuis cette époque, nous avons été amené à préciser davantage la significaction des termes métaphysique et science."19 (1969: 98). La metafísica se dirige al conocimiento de lo absoluto entrando en la cosa, la ciencia, dice, gira en torno a la cosa y es, por tanto, el conocimiento de lo relativo. Pero, esa cosa a la que se dirigen metafísica y ciencia es fundamentalmente movimiento, entendido como duración pura que "...exclut toute idée de juxtaposition, d'extério- rité réciproque et d'étendue. ${ }^{\prime 20}$ Con lo cual dice

\footnotetext{
19 "Desde entonces nos hemos visto obligados a precisar la significación de los términos metafísica y ciencia." (Bergson, 1960: 7).

20 "...excluye toda idea de yuxtaposición, de exterioridad recíproca y de extensión."
} 
más adelante, se advierte que el conocimiento del mundo exterior está determinado por el trabajo de la conciencia que es capaz de captar la movilidad pura, toda vez que se libera del espacio que contiene al movimiento. De esta manera, el sujeto tiene, a la vez,"... une image plus fidèle de notre développement dans la durée. $»^{21}$ (1969: 102) Interioridad y exterioridad se manifiestan en el mismo acto cognoscente que ha clausurado la diferencia entre el mundo exterior y los procesos internos a partir de la noción de duración. En la clausura se afirma la continuidad y la unidad del ser, podría concluirse. Con ello, Bergson afirma que la intuición da la pauta para afirmar que la duración es constitutiva del sujeto y de la totalidad del ser (1969: 103).

A partir de ello, surgen dos cuestiones: ¿qué es el conocimiento? y, ¿qué le corresponde a la ciencia y qué a la metafísica? La conciencia conoce pero no hay dos momentos iguales, luego, cada vez se conoce algo distinto; asimismo, absoluto e infinito se identifican en la medida que, la intuición o simpatía lo captan en la duración; no así el análisis que se remite a los elementos ya conocidos del objeto. En este punto la distancia con Kant es notable pues, "Analyser consiste donc à exprimer une chose en fonction de ce qui n'est pas elle... se conti- nue donc à l'infini. Mais l'intuition, si elle est possible, est un acte simple. 122 (Bergson, 1969: 100). El análisis remite a la ciencia positiva, precisamente a la ciencia que Kant trata de fundamentar. En Los principios metafísicos de la ciencia de la naturaleza (1991), el filósofo alemán define la ciencia como un sistema que reúne la totalidad de conocimientos ordenados por principios enlazados empírica o racionalmente; esta distinción se traduce en dos grupos de ciencias: en el primero está la matemática cuya certeza es apodíctica y la metafísica o filosofía pura, en el segundo están la química y la psicología. (1991: 3-5) Tanto matemática como metafísica se constituyen de igual manera, pero la primera lo hace a través de conceptos construidos intuitivamente, $y$ la segunda, por simples conceptos. Ahora bien, en cuanto al segundo grupo de ciencias, Kant sostiene que la ciencia natural supone una metafísica de la naturaleza en la medida en que ". . la existencia no se puede representar en ninguna intuición a-priori..." (1991: pág. 6), por lo que la metafísica de la naturaleza tratará de las leyes generales que posibilitan el concepto de naturaleza o de cosa que es dada empíricamente; de ello se sigue que, para Kant, conocer la cosa apriori, es "... conocerla por su simple posibilidad..." es decir que, conocer los objetos naturales implica, además de las

\footnotetext{
21 "... una imagen más fiel de nuestro desarrollo en la duración" (Bergson, 1960: 14).

22 "Analizar consiste expresar una cosa en función de lo que no es... se prolonga hasta el infinito. La intuición, si ella es posible, es un acto simple" (Bergson, 1960: 11).
} 
leyes dadas por la metafísica de la naturaleza, conocimientos que surgen fuera del pensamiento (1991: 7). En el ámbito de la ciencia, Kant no podría entender el trabajo de la intuición sensible desligado de las categorías a-priori; con ello, la intuición es algo completamente diferente, tanto en su definición como en su proceso, de lo señalado Bergson. Aún más, las categorías a-priori se definen, dentro de la filosofía kantiana, como determinaciones de nuestra conciencia que imitan las funciones lógicas de los juicios, son, por tanto, "... simples formas de los juicios en tanto que aplicadas a las intuiciones... siempre sensibles." (1991: 11). La objeción de Bergson se da en términos de la duración que capta el movimiento y el cambio permanente que produce; en tal sentido, el conocimiento se enfoca en el mundo, no de las cosas y del doble aspecto que les asigna Kant -empírico y referido al noúmeno-, sino de la movilidad pura. El resultado del conocimiento no se ciñe al concepto, lo sobrepasa, llega a la esencia del mundo porque da cuenta también de la vida interior y de su propio movimiento:

La vie intérieure est tout cela à la fois, va- riété de qualités, continuité de progrès, unité de direction. On ne saurait la représenter par des images.

Mais on la représenterait bien moins encore par des concepts, c'est-à-dire par des idées abstraites, ou générales, ou simples. Sans doute aucune image ne rendra tout à fait le sentiment original que j'ai de lécoulement de moi-même... À celui qui ne serait pas capable de se donner à lui-même l'intuition de la durée constitutive de son être, rien ne la donneraitjamais, pas plus les concepts que les images. ${ }^{23}$ (Bergson, 1969: 102).

La vida interior así concebida implica, según Russell, que"...the time here spoken of is not mathematical time, the homogeneous assemblage of mutually external instants. Mathematical time, according to Bergson, is really a form of space; the time which is of the essence of life is what he calls duration."24 (1946: 823). En la misma línea de Russell Kant considera que en el espacio no hay nada móvil y el tiempo es"... la forma real de la intuición interna" (2006: 82), no tiene en sí cambio alguno; las dos categorías están ligadas a la experiencia en tanto condiciones que la posibilitan.

\footnotetext{
23 "La vida interior es todo esto a la vez variedad de cualidades, continuidad de progreso, unidad de dirección. No podría representársela por imágenes.

Pues menos aún se la representaría por conceptos, esto es, por ideas abstractas, o generales, o simples. Sin duda ninguna imagen expresará completamente el sentimiento original que yo tengo de la fluencia de mí mismo... Al que no sea capaz de darse a sí mismo la intuición de la duración constitutiva de su ser, nada se la dará jamás, ni los conceptos ni la imágenes." (Bergson, 1960: 15).

24 "...el tiempo del que aquí se habla no es el tiempo matemático, la reunión homogénea de instantes mutuamente externos. El tiempo matemático, de acuerdo a Bergson, es realmente una forma del espacio; el tiempo que es la esencia de la vida es lo que él llama duración."
} 
El concepto que completa el sistema bergsoniano, según Russell, es el de memoria; es en ella donde se muestra la duración, en tanto en la memoria el pasado se mantiene en el presente. Entonces se determina que "Duration is the very stuff of reality, which is perpetual becoming, never something made."25 (1946: 824). En la memoria confluyen mente y materia, pero ello implica una problemática que recae sobre la acción del sujeto que se halla en tensión al tratar de reunir el pasado que está deslizándose, en un todo que es el presente, dice Russell. Pero lo que resulta realmente problemático en este punto, es la oposición que Bergson plantea entre memoria y percepción pura; esta última, en el acto de conocer, se convierte casi en materia; pues, la percepción sin memoria no logra captar la duración. Estas consideraciones, sin embargo, dan cuenta del interés del filósofo francés por los procesos del cerebro que se ocupan, según dice, solo de dar paso a lo útil, de lo cual resulta un sujeto que fluctúa entre esos procesos y los de la memoria; el sistema bergsoniano se dirige a dilucidar los contenidos de la duración, mismos que remiten a la realidad verdadera. La explicación de la oposición entre memoria y percepción se da con relación a otra oposición más fundamental, la que se da entre instinto e intelecto. Podría decirse que el instinto constituye a la intuición y el intelecto está en el orden de la percepción. En este contexto Bergson señala que el intelecto da cuenta del espacio en tanto que la intuición del tiempo, de este modo, el mundo de las cosas se diluye y solo quedan las acciones.

En Matière et mémoire( ), Bergson se refiere a la diferencia con Kant :

Limpuissance de la raison spéculative, telle que Kant l'a démontrée, n'est peutêtre, au fond, que l'impuissance d'une intelligence asservie à certaines nécessités de la vie corporelle et s'exerçant sur une matière qu'il a fallu désorganiser pour la satisfaction de nos besoins. Notre connaissance des choses ne serait plus alors relative à la structure fondamentale de notre esprit, mais seulement à ses habitudes superficielles et acquises, à la forme contingente quill tient de nos fonctions corporelles et de nos besoins inférieurs. ${ }^{26}$ (2012 : 205).

\footnotetext{
25 "La duración es el verdadero tejido de la realidad, que está perpetuamente deviniendo y nunca es algo hecho."

${ }^{26}$ La impotencia de la razón especulativa, como Kant lo ha demostrado, no es, en el fondo, más que la impotencia de una inteligencia esclavizada por ciertas necesidades de la vida corporal y ejerciéndose sobre una materia a la que ha necesitado desorganizar para la satisfacción de nuestras necesidades. Nuestro conocimiento de las cosas ya no sería entonces relativo a la estructura fundamental de nuestro espíritu, sino solamente a sus hábitos superficiales y adquiridos, a la forma contingente que se atiene a nuestras funciones corporales y de nuestras necesidades inferiores (Bergson, 2013: 194).
} 
Con ello quiere significar que la razón especulativa kantiana no logra determinar los aspectos que dan cuenta de la realidad en movimiento; de hecho Kant postula mediaciones entre las cosas y el conocimiento, estas mediaciones son los fenómenos, producto de una forma de interpretar las relaciones del sujeto con el mundo exterior, interpretación en la que el espacio y el tiempo devienen como formas puras de la intuición sensible; esta caracterización del espacio y el tiempo tienen un interés especulativo y no vital, que termina por colocar el problema del conocimiento en el idealismo. Las consecuencias del idealismo alcanzan tanto al proceso del conocimiento como a la ciencia: "Qu'on y regarde de près: on verra que l'écueil de tout idéalisme est là; il est dans ce passage de l'ordre qui nous apparaît dans la perception à l'ordre que nous réussit dans la science - ou, s'il s'agit plus particulièrement de l'idéalisme kantien, dans le passage de la sensibilité à l'entendement. » ${ }^{27}$ (Bergson, 2012: 255). La crítica de Bergson no alcanza solamente al kantismo sino también al racionalismo y al empirismo; en la Introducción a la metafísica (1960), cuando trabaja el concepto de análisis, señala que este ha sido confundido con la ciencia y la metafísica. Los términos de la confusión se hallan en la psicología experimental que busca determinar es- tados psicológicos para dar cuenta del yo. Este camino resulta en una inconsistencia pues, se busca, a través del análisis, la interioridad del sujeto que, como se ha visto, solo puede ser captada por la intuición, intuición que se opone al análisis. Ahora bien, estas críticas le permiten a Bergson delimitar la metafísica que sostiene tanto a empiristas como a racionalistas y que consiste en ir de los conceptos a la realidad, más aún, el problema de esta metafísica consiste en la aplicación de procedimientos, dirigidos a encontrar conocimientos de utilidad práctica, en la búsqueda de lo real (Kant I., 1991: 36). Resulta interesante que, al igual que Kant, Bergson encuentre que la forma de constituir conocimientos de la metafísica es compartida por las ciencias y que, en ello, la ciencia halla su principal problema. Sin embargo, en eso mismo que deviene como similar, puede determinarse una diferencia entre los dos filósofos; a saber, para Kant, el concepto de metafísica se formula sintéticamente a partir de principios y de la investigación en la razón pura y en sus leyes; este trabajo, sin embargo, se sustenta en un ejercicio previo que muestra lo que se debe hacer para tener una ciencia de la realidad, es decir, define un método cuyo momento central será analítico, en este sentido dice,"... se parte delo que se investiga... y se asciende a las

\footnotetext{
${ }^{27}$ Miremos esto de cerca: se verá que el escollo de todo idealismo está ahí; está en el pasaje del orden que nos aparece en la percepción al orden que obtenemos en la ciencia -o si se trata del idealismo kantiano, en el pasaje de la sensibilidad al entendimiento (Bergson, 2013: 235).
} 
condiciones que... lo posibilitan." (Kant, 1980: 72) El error para Kant, en la metafísica anterior, ha sido no poner en cuestión la razón y sus límites para obrar correctamente; es necesaria la filosofía trascendental que precede a la metafísica y a la ciencia, tanto a la matemática pura, como a la ciencia natural; estas ciencias teóricas representan los objetos en la intuición a partir de las categorías a-priori. (1980: 76) Toda esta empresa supone el trabajo de la razón como fuente y posibilidad del conocimiento. Para Bergson, el error de Kant está en atribuir a la intuición categorial, fundamentada solamente en la razón y en su carácter lógico, la condición y posibilidad de la metafísica y de la ciencia. Este error se traduce en la pérdida del objeto de la ciencia que confunde"...l'appareil logique de la science pour la science même, oubliant l'intuition d'où le reste a pu sortir." 28 (1969: 118) Aquello que permite delimitar el campo de la ciencia del campo de la metafísica es la intuición pero, en cada autor, la intuición se define en un contexto distinto.

\section{CONCLUSIONES}

Dentro de los límites de este artículo, la relación que se ha construido entre Bergson y Kant, en parte mediante Russell y Merleau-Ponty, ha centrado la discusión en los alcances del concepto de intuición que el filósofo francés define respecto del criticismo kantiano. El trabajo de Russell, profundamente arraigado en la tradición positivista, entiende que Bergson se ubica en una suerte de irracionalismo negador de los principios del conocimiento y del trabajo científico; Merleau-Ponty, al contrario, determina que el bergsonismo abre una nueva ruta para la ciencia en la medida que introduce en el entendimiento potencias complementarias que permiten al sujeto adentrarse en el ámbito del mundo que Kant había vedado para la razón pura.

En la línea de esta reflexión es posible dilucidar el sentido de la duración, categoría que permite a Bergson considerar la realidad en movimiento y, al sujeto con la capacidad de conocerla en su verdad. Esta concepción tiene consecuencias tanto en el plano del conocimiento como en el de la ontología. La realidad deviene dinámica, imposible de ser captada por el entendimiento racional; en el proceso que esto implica, la inteligencia actúa a profundidad superando a la intuición que solo capta el mundo material. En este punto, la inteligencia deviene creadora pues hay otra

28 "... el aparato lógico de la ciencia por la ciencia misma... olvidando la intuición de donde todo ha podido salir." (Bergson, 1960: 38 -39). 
intuición, que es capaz de atrapar la esencia de lo real, esto es su movimiento. De esta manera, el entendimiento que en Kant actúa a-priori, con estructuras lógico-formales para aprehender el mundo exterior, queda limitado al dato sensible sin dar cuenta de la esencialidad que presupone Bergson.

La representación del cambio y del movimiento, en la filosofía bergsoniana, llevará a un orden distinto para definir espacio y tiempo, distinto al orden kantiano que los define como a-priori, es decir, como condiciones de toda percepción posible. Bergson dirá que entre nosotros y el cambio hay un velo, el tiempo es el cambio, la verdad de lo real $y$, el espacio es el velo que cubre la verdad. Esta distancia entre los dos filósofos da a la ciencia y a la metafísica condiciones de posibilidad que resultan diversas; en la filosofía bergsoniana la metafísica es posible pero desde el conocimiento que surge de la intuición que capta el absoluto, dado en términos de movimiento; en la filosofía kantiana, la posibilidad de construir conocimiento a-priori, que reside en el entendimiento del sujeto y, el análisis de los límites de la razón, harán posible la metafísica.

Este aspecto importante para los dos autores gira en torno a la ciencia y su naturaleza. Kant fundamenta la ciencia en la capacidad del sujeto de construir juicios sintéticos a-priori cuya certeza es apodíctica, entiende así, que la ciencia por excelencia es la Matemática. Bergson, crítico de la concepción kantiana de entendimiento -excesivamente independiente de la realidad verdadera- dirá que la ciencia es el resultado del pensamiento creador que remite a los actos de la conciencia no fundados en los datos de los sentidos sino la actividad propia de la realidad. 


\section{BIBLIOGRAFÍA}

Bergson, H. (1960). Introducción a la metafísica. México: UNAM.

Bergson, H. (1963). La evolución creadora. Madrid: Aguilar.

Bergson, H. (1969). Le pensée et le mouvant Essais et conféfences. Paris: Les Presses Universitaires de France.

Bergson, H. (1972). El pensamiento y lo moviente. Buenos Aires: La Pléyade.

Bergson, H. (1999). Ensayo sobre los datos inmediatos de la conciencia. (J. M. Palacios, Trad.) Salamanca: Sígueme.

Bergson, H. (14 de Octubre de 2003). L'évolution créatrice. Québec, Canadá.

Bergson, H. (2011). Essai sur les données immédiates de la conscience (9 ed.). Paris: PUF.

Bergson, H. (2012). Matière et mémoire (9 ed.). Paris: PUF.
Bergson, H. (2013). Materia y memoria. Buenos Aires: Cactus.

Kant, I. (1980). Prolegómenos a toda metafísica futura. Buenos Aires: Aguilar.

Kant, I. (1991). Principios metafísicos de la ciencia de la naturaleza. Madrid: Tecnos.

Kant, I. (2006). Crítica de la razón pura. (P. Ribas, Trad.) México: Taurus.

Merleau-Ponty, M. (1964). Signos. Barcelona: Seix Barral.

Russell, B. (1946). History of Western Philosophy. s.c.: Unwin Brothers Limited.

Worms, F. (2001). L'intelligence gagnée par l'intuition. Les études philosophiques, 453464. 